\title{
Lymphoma Response to Immunomodulatory Therapy Criteria Indeterminate Response 1
}

National Cancer Institute

\section{Source}

National Cancer Institute. Lymphoma Response to Immunomodulatory Therapy Criteria Indeterminate Response 1. NCI Thesaurus. Code C153153.

An increase in overall tumor burden of greater than or equal to 50 percent of up to six measurable lesions in the first 12 weeks of therapy, without clinical deterioration. 